\title{
Algebraic Geometric Solutions of Einstein's Equations: Some Physical Properties
}

\author{
D. A. Korotkin \\ Department of Mathematics, Leningrad Institute of Aircraft Instrumentation, \\ Gertzena, 67, SU-190000 Leningrad, USSR
}

Received June 14, 1990; in revised form October 29, 1990

\begin{abstract}
The physical properties of algebraic geometric solutions of stationary axisymmetric vacuum Einstein's equations are discussed. It appears that these solutions describe an interaction of a few localized rotating string-like objects on an arbitrary static background. If such an object collapses to a point then it produces a Kerr-NUT black hole.
\end{abstract}

\section{Introduction}

Recently the author and V.B. Matveev have shown [1-3] that the technique of multidimensional theta-functions first used by S.P. Novikov, B.A. Dubrovin, V.B. Matveev, A.R. Its, and I. M. Krichever [4-6] for finding periodic and almost periodic solutions of Korteveg de Vries (KdV), Non-linear Schroedinger (NSch) and other equations solvable by the inverse scattering method may be successfully applied to a stationary axisymmetric Einstein's equation in a vacuum and to the Einstein-Maxwell system. Further in [7,8] the technique of [1-3] was extended to the four-dimensional self-duality equations for $S U(2)$ and $S U(1,1)$ groups.

The main properties of finite-gap solutions from [1-3] differ radically from the properties of well-known finite-gap periodic and almost periodic solutions of $\mathrm{KdV}$, NSch and others. Namely the dynamics in finite-gap solutions of Einstein's equations is set by the deformation of the algebraic curve with variable branch points in contrast to the dynamics of the usual finite-gap solutions set by the linear flow on the Jacobi manifold of the fixed curve. As a consequence the solutions from [1-3] are not periodic but localized as degenerated-soliton solutions. It appeared possible to introduce in these solutions the functional parameters corresponding to an arbitrary static background.

This paper is devoted to the investigation of most elementary physical properties of non-degenerated finite-gap solutions of vacuum stationary axisymmetric Einstein's equation found in [1-3]. The general genus $g$ solution of stationary axisymmetric Einstein's equation describes an interaction of $g$ localised 
string-like objects on an arbitrary static non-rotating background. Section 1 includes some general facts and a brief description of the algebraic geometric construction of the solutions based on [1-3]. In Sect. 2 we show that the multi-gap solution on the flat background may be considered as an interaction of a few localized genus 1 objects. In Sect. 3 we consider the simplest genus 1 solution and obtain some results about its topology, mass, angular momentum, NUTparameter, singularities and infinite redshift surface in the case of a vertical immovable branch cut. We distinguish effectively a subclass of solutions with a vanishing NUT-parameter. Section 4 is devoted to some concluding remarks.

\section{Algebraic-Geometric Solutions of Stationary Axisymmetric Vacuum Einstein's Equation}

We will write the line element of stationary axisymmetric space in the following widely used form:

$$
d s^{2}=f^{-1}\left[e^{2 k}\left(d \varrho^{2}+d z^{2}\right)+\varrho^{2} d \varphi^{2}\right]-f(d t+A d \varphi)^{2},
$$

where $(\varrho, z)$ are Weyl coordinates; functions $f, k$, and $A$ may be expressed in terms of one complex valued function - the Ernst potential $\mathscr{E}(\varrho, z)$ :

$$
f=\operatorname{Re} \mathscr{E}, \quad A_{\zeta}=2 \varrho \frac{(\mathscr{E}-\overline{\mathscr{E}})_{\zeta}}{(\mathscr{E}+\overline{\mathscr{E}})^{2}}, \quad k_{\zeta}=2 \varrho \frac{\mathscr{E}_{\mathscr{E}} \overline{\mathscr{E}}_{\zeta}}{(\mathscr{E}+\overline{\mathscr{E}})^{2}},
$$

where $\zeta=\varrho+i z$ and $\bar{\zeta}=\varrho-i z-$ new complex coordinates.

The Einstein's equation in terms of $\mathscr{E}(\zeta, \bar{\zeta})$ may be written in the Ernst form

$$
(\mathscr{E}+\overline{\mathscr{E}})\left(\mathscr{E}_{z z}+\varrho^{-1} \mathscr{E}_{\varrho}+\mathscr{E}_{\varrho \varrho}\right)=2\left(\mathscr{E}_{z}^{2}+\mathscr{E}_{\varrho}^{2}\right)
$$

The construction of finite-gap solutions of (1.3) based on the zero curvature representation found in [9] is as follows [1-3]:

Let's consider the hyperelliptic Riemann surface $\mathscr{L}$ of genus $g$ (Fig.1) depending on $(\zeta, \bar{\zeta})$ and defined by the equation

$$
\omega^{2}=(\lambda-i \bar{\zeta})(\lambda+i \zeta) \prod_{i=r}^{g}\left(\lambda-E_{i}\right)\left(\lambda-F_{i}\right)
$$

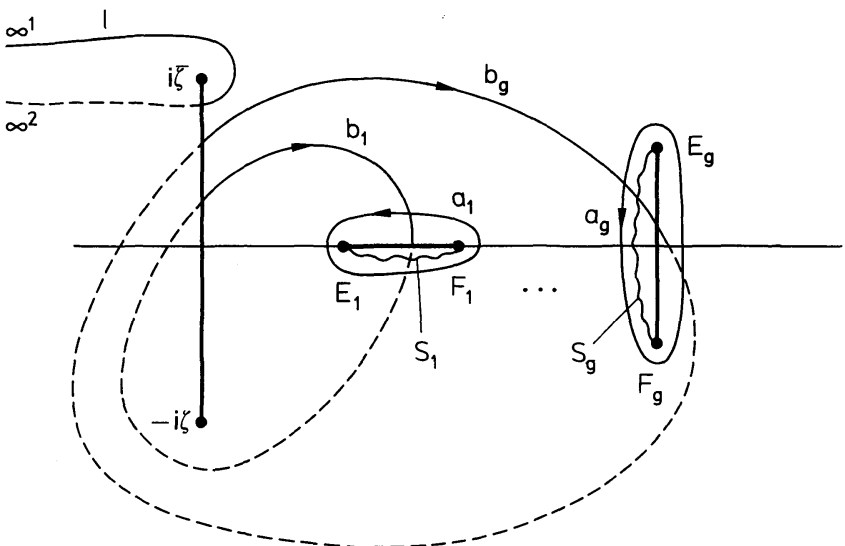

Fig. 1. Hyperelliptic curve $\mathscr{L}$. Continuous contours lie on the $1^{\text {st }}$ sheet, dotted-on the $2^{\text {nd }}$ 
where $E_{i}, F_{i}, i=1, \ldots, g$ are independent of $\zeta, \bar{\zeta}$. This curve must be real, i.e. $\forall i=1, \ldots, g, E_{i}=\bar{F}_{i}$ or $E_{i}, F_{i} \in \mathbb{R}$. Canonical algebraic-geometric objects on $\mathscr{L}$ follow: the basis of cycles $\left(a_{i}, b_{i}\right)$ being chosen as is shown in Fig. 1; dual basis of holomorphic 1-forms $d U_{j}(P), j=1, \ldots, g$ with the normalization $S_{a_{i}} d U_{j}=\delta_{i j}$; Abel mapping $U_{j}(P)=S_{P_{0}}^{P} d U_{j}, P, P_{0} \in \mathscr{L}, j=1, \ldots, g$. Matrix $\mathbb{B}_{i j}=S_{b_{i}} d U_{j}, i, j=1, \ldots, g$ is a matrix of $b$-periods associated with $\mathscr{L}$.

The Riemann theta-function is defined by the following series:

$$
\theta(z \mid \mathbb{B})=\sum_{m \in \mathbb{Z}^{g}} \exp (\pi i\langle\mathbb{B} m, m\rangle+2 \pi i\langle z, m\rangle),
$$

where $z \in \mathbb{Z}^{g},\langle.,$.$\rangle - ordinary scalar product.$

For a detailed description of the properties of ordinary algebraic geometric objects associated with $\mathscr{L}$, see [2].

The finite-gap solution of (1.3) found in [1-3] is written in the following form:

$$
\begin{aligned}
\mathscr{E}(\zeta, \bar{\zeta})= & \frac{\theta\left(U\left(\infty^{2}\right)-U(D)-K+n / 4+b \mid \mathbb{B}\right) \theta\left(U\left(\infty^{1}\right)-U(D)-K \mid \mathbb{B}\right)}{\theta\left(U\left(\infty^{1}\right)-U(D)-K+n / 4+b \mid \mathbb{B}\right) \theta\left(U\left(\infty^{2}\right)-U(D)-K \mid \mathbb{B}\right)} \\
& \times \exp \left\{\left.\left[W_{0}(P)+W(P)\right]\right|_{\infty^{1}} ^{\infty^{2}}\right\}
\end{aligned}
$$

where $K$ is a vector of Riemann constants; $\infty^{1,2}$ are infinite points on the first and second sheets of $\mathscr{L} ; n_{k}= \pm 1, k=1, \ldots, g ; D=D_{1}+\ldots+D_{g}$ is an arbitrary nonspecial divisor independent of $(\zeta, \bar{\zeta})\left(D_{i} \in \mathscr{L}\right)$ satisfying the reality condition $D^{\tau}=D$, where $\tau$ is a conjugation anti-involution on every sheet of $\mathscr{L}$. For the abelian integral $W_{0}(P)$ we have

$$
W_{0}(P)=\frac{1}{2} \sum_{k=0}^{g} n_{k} W_{E_{k}, F_{k}}(P),
$$

where $W_{Q, R}(P)$ is a third kind integral with the residue +1 at point $Q$ and -1 at $R$; cuts $s_{k}$ between $E_{k}$ and $F_{k}$ are shown in Fig. 1. The abelian integral $W(P)$ with a vector of $b$-periods $2 \pi i b$ is an arbitrary linear combination of abelian integrals of the second and the third kind; all singularities of these integrals and coefficients of the linear combination are independent of $(\zeta, \bar{\zeta})$. The integral $W(P)$ must satisfy the following reality conditions: $\left.W(P)\right|_{\infty^{1}} ^{\infty^{2}} \in \mathbb{R}$ and $2 \pi i b \in \mathbb{R}$. These conditions impose some restrictions on singularities of $W(P)$ and coefficients of the linear combination; for details see $[1,2]$.

The integral $W(P)$ in (1.5) corresponds to the static background. Indeed, if we take $g=0$ then $\mathscr{E}(\zeta, \bar{\zeta})=\exp \left\{\left.W(P)\right|_{\infty_{1}^{1}} ^{\infty^{2}}\right\} \in \mathbb{R}$ and (1.3) we may be rewritten as $\Delta(\ln \mathscr{E})=0$, where $\Delta=\partial_{\varrho}^{2}+\varrho^{-1} \partial_{\varrho}+\partial_{z}^{2}$. This linear equation gives solutions of the static (Weyl) class.

If we consider an infinite limit in the linear combination setting $W(P)$ then we obtain a solution including some functional parameters. We see that there is a close link between the linearity of the Ernst equation for the static solutions and the existence of the linear structure on the manifold of integrals $W(P)$.

Dependence of $\mathscr{E}$ on $\zeta$ and $\bar{\zeta}$ is given by the dependence of $\mathbb{B}, U\left(\infty^{1,2}\right)-U(D)$, $\left.W(P)\right|_{\infty_{1}^{1}} ^{\infty^{2}}$ and $K$ on $\zeta$ and $\zeta$, i.e. by the movement of our curve $\mathscr{L}$ with fixed divisor $D$ and singularities of $W(P)$ in the moduli space.

To obtain from (1.5) the multi-Kerr-NUT solution on flat background we can choose

$$
W(P)=\sum_{k=1}^{g} 2 i \alpha_{k} W_{E_{k} F_{k}}(P)
$$


where $\alpha_{k} \in \mathbb{R}$ is independent of $(\zeta, \bar{\zeta})$. Cuts $s_{k}$ between $E_{k}$ and $F_{k}$ and the contour $l$ between $\infty^{1}$ and $\infty^{2}$ (Fig. 1) are chosen such that $\left.W(P)\right|_{\infty} ^{\infty^{2}}=0$ (i.e. the background is flat indeed) and $b_{k}=i \alpha_{k}$.

Let's consider a limit $E_{k}, F_{k} \rightarrow \lambda_{k} \in \mathbb{R} . k=1, \ldots, g$. In this limit $\mathscr{L}$ degenerates into a surface of genus zero and $\mathscr{E}(\zeta, \zeta)$ may be expressed in elementary functions; it describes an interaction of $g$ Kerr-NUT objects.

However, $4 g$ physical parameters of this solution: masses, angular momentum, sites on the axis and NUT-parameters, are set only by $3 g$ real parameters: $D_{i}, \lambda_{i}$, and $a_{i}$. To obtain a general family of multi-Kerr-NUT solutions it is necessary to take $g=2 \tilde{g}$ and $D_{i}=D_{i+\tilde{g}}^{*}, i=1, \ldots, \tilde{g}$, where $*$ is an involution on $\mathscr{L}$ interchanging the sheets. Then (1.5) describes an interaction of $\tilde{g}$ Kerr-NUT objects with the horizons dependent on $4 \tilde{g}$ real parameters: $\lambda_{1}, \ldots, \lambda_{2 \tilde{g}}$ and $a_{1}, \ldots, a_{2 \tilde{g}}$. If some pair $\left(\lambda_{i}, \lambda_{i+g}\right)$ is not real but $\lambda_{i}=\bar{\lambda}_{i+g}$, then the corresponding Kerr-NUT component has a naked singularity instead of the horizons.

\section{Asymptotic Properties of Finite-Gap Solutions}

Here we will show that in some sense the finite-gap solution may be considered as an interaction of a few localized one-gap solutions and a static background set by $W(P)$. Asymptotic behaviour of the finite-gap solution is defined in zero approximation only by static background, i.e. the asymptotic properties of finite-gap solutions coincide with the properties of degenerated-multi-KerrNUT solutions on static background.

We shall use in this paper the expression "asymptotic flatness" in two senses. The solution will be called asymptotically flat in a weak sense if the Ernst potential tends to 1 on the infinity; if also the mass of the object is real, then the solution will be called asymptotically flat in a strong sense.

At first we shall show that one-gap solution on a flat background is asymptotically flat in a weak sense if contours $s$ and $l$ are chosen as shown in Fig. 1 (for $g=1$ ). Let $\beta=\zeta / \zeta \mid$; consider a limit $|\zeta| \rightarrow \infty$ with $\beta=$ const. Solution (1.5) is invariant under the similarity transformation of $\mathscr{L}$ with the pole divisor; let's define by $\mathscr{L}^{\prime}$ the result of this transformation with the centre in 0 and coefficient $1 /|\zeta|$.

In the limit $|\zeta| \rightarrow \infty$ curve $\mathscr{L}^{\prime}$ degenerates into a zero genus curve. Therefore we can apply to this case the results of $[1,2]$ about the behaviour of all algebraic geometric objects in this limit:

$$
\begin{gathered}
\operatorname{Re}(i \mathbb{B})=-\frac{1}{\pi} \ln |\zeta|+O(1), \\
U\left(\infty^{1,2}\right)-U(D)-K \equiv U\left(\infty^{1,2}\right)-U(D)-\frac{\mathbb{B}+1}{2}=O(1),
\end{gathered}
$$

and

$$
\begin{gathered}
\theta\left(U\left(\infty^{1,2}\right)-U(D)-K\right) \underset{|\xi| \rightarrow \infty}{\longrightarrow} 1, \\
\theta\left(U\left(\infty^{1,2}\right)-U(D)-K+\frac{1}{4}\right) \underset{|\xi| \rightarrow \infty}{\longrightarrow} 1 .
\end{gathered}
$$

So $\mathscr{E}$ tends to 1 in any direction and one-gap solution on the flat background is asymptotically flat in a weak sense. 
In a full analogy we can prove the same fact for a $g$-gap solution when $g>1$ and the contour $l$ doesn't cross the contours $s_{i}, i=1, \ldots, g$. If contour $l$ crosses some of the contours $s_{i}$ then the factor $\exp \left\{W_{0}\left(\infty^{1}\right)-W_{0}\left(\infty^{2}\right)\right\}$ independent of $(\varrho, z)$ may be not equal to 1 . In this case the asymptotic value of the Ernst potential is equal to the value of this factor. Therefore our choice of contour $l$ corresponds to one of the asymptotically flat in a weak sense ("physical") sheets of the manifold set by the solution (1.5) in the case $W(P)=0$.

Let's consider the genus $g$ solution with $W(P)=0$ when the distances between branch cuts $\left[E_{i}, F_{i}\right]$ are large in comparison with the lengths of the cuts. Let also point $D_{i}$ be situated in the neighbourhood of the cut $\left[E_{i}, F_{i}\right], i=1, \ldots, g$. More exactly, let's consider a limit $\xi \rightarrow \infty$ when

$$
\begin{aligned}
E_{i} & =\xi \varkappa_{i}+e_{i}, \\
F_{i} & =\xi \varkappa_{i}+f_{i}, \quad \varkappa_{i} \neq \varkappa_{j}, \quad i, j=1, \ldots, g, \xi \in \mathbb{R}, \\
D_{i} & =\xi \varkappa_{i}+d_{i} .
\end{aligned}
$$

Consider again a similarity transformation of $\mathscr{L}$ with the coefficient $\xi^{-1}$-curve $\mathscr{L}^{\prime \prime}$. We intend to investigate the behaviour of this solution in the neighbourhood of the $i^{\text {th }}$ cut when $\zeta=E_{i}+O(1)$ (in the limit $\xi \rightarrow \infty$ ). In this limit curve $\mathscr{L}^{\prime \prime}$ degenerates, all branch cuts tend to points; branch cuts $[i \zeta, i \bar{\zeta}]$ and $\left[E_{i}, F_{i}\right]$ are also glued.

The behaviour of the basic holomorphic differential $d U_{k}^{\prime \prime}(P)$ on $\mathscr{L}^{\prime \prime}$ is the following:

$$
\begin{gathered}
d U_{k}^{\prime \prime}(P) \underset{\zeta \rightarrow \infty}{\longrightarrow} \frac{1}{2 \pi i} \frac{d \lambda}{\lambda-\varkappa_{k}}, \quad k \neq i ; \\
d U_{i}^{\prime \prime}(\xi P) \underset{\zeta \rightarrow \infty}{\longrightarrow} d U_{i}^{\circ}(P),
\end{gathered}
$$

where $d U_{i}^{\circ}(P)$ is a normalized abelian differential of the first kind on a genus one surface $\mathscr{L}_{i}$ with branch cuts $[-i \zeta, i \bar{\zeta}]$ and $\left[E_{i}, F_{i}\right]$.

Therefore the elements of the matrix $\mathbb{B}$ (the same for $\mathscr{L}$ and $\mathscr{L}^{\prime \prime}$ ) are:

$$
\begin{gathered}
\mathbb{B}_{i i}=\mathbb{B}_{i}+o(1) ; \\
\operatorname{Re}\left(i \mathbb{B}_{j j}\right)=\frac{1}{\pi} \ln \xi+O(1), \quad j \neq i ; \\
\mathbb{B}_{j k}=O(1), \quad j, k=1, \ldots, g, \quad j \neq k, \xi \rightarrow \infty,
\end{gathered}
$$

where $\mathbb{B}_{i}$ is a $1 \times 1$ matrix of $b$-periods of curve $\mathscr{L}_{i}$.

For the Abel mapping of the divisor $D$ on $\mathscr{L}$ we have:

$$
\begin{gathered}
U_{i}\left(D_{i}\right)=U_{i}^{\circ}\left(D_{i}\right)+o(1), \\
U_{j}\left(D_{j}\right)=\frac{1}{2} \mathbb{B}_{j j}+O(1), \quad j \neq i, \\
U_{k}\left(D_{j}\right)=O(1), \quad j \neq k, \quad j, k=1, \ldots, g .
\end{gathered}
$$

Substituting (2.1) and (2.2) into (1.5) and using the definition of the thetafunction it is easy to obtain

$$
\mathscr{E}(\zeta, \bar{\zeta}) \underset{\left|\zeta-E_{i}\right|=O(1)}{\longrightarrow} \mathscr{E}_{i}(\zeta, \bar{\zeta}),
$$

where $\mathscr{E}_{i}(\zeta, \bar{\zeta})$ is a genus 1 potential corresponding to curve $\mathscr{L}_{i}$ with the divisor $D_{i}$. 
So the genus $g$ solution on a flat background in the limit $\xi \rightarrow \infty$ splits into $g$ localized genus one components; their interaction vanishes if the distances between the components tend to infinity, but their sizes remain constant.

Note that we proved rigorously this statement only for identical "splitting rates" of the branch cuts and corresponding points of the divisor. However, this assumption was introduced only for convenience and the previous statement may be easily proved in the general case too.

Let now $W(P) \neq 0$, i.e. the static background is not flat. In full analogy with the case $W(P)=0$ we can check that the rotating one-gap objects are localized; as a consequence, the behaviour of $\mathscr{E}(\zeta, \bar{\zeta})$ on infinity is set [with the precision $o(1)]$ by the static background.

So to obtain a more or less complete physical interpretation of the genus $g$ solution it is necessary to investigate the elementary genus 1 solution.

\section{Analysis of Some Physical Properties of the Genus 1 Solution}

In this section we shall consider the simplest asymptotically flat in a weak sense (on the "physical" sheet) genus one solution. The main focus will be on the case of a vertical immovable branch cut on an associated algebraic curve (Fig. 2), because this case seems more transparent from the physical point of view in comparison with the case of a horizontal immovable cut. For both cases we discuss the behaviour of the solution on the symmetry axis, its mass, angular momentum and NUT-parameter. For the "vertical" case we investigate also the topology, infinite redshift surface, singularities and some other properties.

If $g=1$ and the background is flat then formula (1.5) may be written as

$$
\begin{aligned}
\mathscr{E}(\zeta, \bar{\zeta})= & \frac{\theta\left(U\left(\infty^{2}\right)-U(D)-K+1 / 4+i \alpha\right) \theta\left(U\left(\infty^{1}\right)-U(D)-K\right)}{\theta\left(U\left(\infty^{1}\right)-U(D)-K+1 / 4+i \alpha\right) \theta\left(U\left(\infty^{2}\right)-U(D)-K\right)} \\
& \times \exp \left\{\left.\left(2 i \alpha+\frac{1}{2}\right)\left[W_{E F}(P)\right]\right|_{\infty^{2}} ^{\infty}\right\},
\end{aligned}
$$

where we introduce a new notation $E_{1}=E, F_{1}=F ; D$ is an arbitrary immovable point of the curve $\mathscr{L}$.

If $\mathscr{L}$ degenerates, i.e. $E, F \rightarrow \gamma \in \mathbb{R}$, solution (3.1) transforms into a threeparametric Kerr-NUT solution with the horizons.

Namely, (see $[1,2])$ if we introduce prolate ellipsoidal coordinates $\left(x_{0}, y_{0}\right)$ :

$$
x_{0}=\frac{1}{D-\gamma}\left[f_{0}(\gamma)+f_{0}(D)\right], \quad y_{0}=\frac{1}{D-\gamma}\left[f_{0}(\gamma)-f_{0}(D)\right],
$$

where $f_{0}(\lambda) \equiv\left(\varrho^{2}+(\lambda-z)^{2}\right)^{1 / 2}$ then (3.1) takes the form

$$
\mathscr{E}_{0}(\varrho, z)=\frac{y_{0}-1+i e^{-2 \pi \alpha}\left(x_{0}+1\right)}{y_{0}+1+i e^{-2 \pi \alpha}\left(x_{0}-1\right)} .
$$

This is a Kerr-NUT solution with NUT-parameter $\operatorname{arctg}\left(e^{2 \pi \alpha}\right)$ and mass $M=\frac{1}{2}(D-\gamma)\left(1+i e^{-2 \pi \alpha}\right)$. Expression (3.3) gives a three-parametric subclass of a general four-parametric Kerr-NUT family. We shall use formulae (3.2) and (3.3) for the investigation of the behaviour of a non-degenerated solution on the symmetry axis. 
Now let's consider a topology of "Ernst manifold" corresponding to the solution (3.1).

\section{A. Topological Structure of Ernst Manifold:}

\section{Case $E=\bar{F}$; Topological Reconstruction in Kerr-NUT Limit}

Due to the presence of two Killing vectors we can consider here the twodimensional section $t=$ const, $\varphi=$ const $\mathfrak{M}$ of the four-dimensional "Ernst manifold" $\mathfrak{\mathfrak { M }}$. So we can use in application to $\mathfrak{M}$ a convenient language of the theory of Riemann surfaces.

Note that due to the integral character of the link (1.2) between the Ernst potential and metric coefficients the Einstein manifold corresponding to our Ernst manifold $\mathfrak{\mathfrak { M }}$ is in the general case a covering of $\mathfrak{\mathfrak { M }}$; the branch points of this covering may be at points where the Ernst equation is not valid.

For $\mathfrak{M}$ to have no boundary it is necessary to consider a symmetrical continuation of $\mathscr{E}(\zeta, \bar{\zeta})$ in the domain of negative $\varrho: \mathscr{E}(-\varrho, z)=\mathscr{E}(\varrho, z)$ (or, equivalently, to consider a section of $\mathfrak{M} t=$ const, $\varphi=$ const $+k \pi, k=0,1)$.

As a result we can consider $\mathfrak{M}$ as a covering of a plane with complex coordinate $i \bar{\zeta}=z+i \varrho \in \mathbb{C}$.

Denote by $\mathscr{L}_{j}^{i}$ (in our case $i=1,2, j \in \mathbb{Z}$ ) the curve $\mathscr{L}$ with some set of branch cuts, basic cycles and contours $\left[\infty^{1}, \infty^{2}\right]$ marked by the pair $\left(\begin{array}{l}i \\ j\end{array}\right)$. We shall mark also by the indexes $i$ and $j$ all objects related to curve $\mathscr{L}_{j}^{i}$. So $\mathscr{E}_{j}^{i}$ is an Ernst potential set by (3.1); $\mathfrak{M}_{j}^{i}$ is a corresponding sheet of the Ernst manifold.

Let's start with the curve $\mathscr{L}_{0}^{1}$ shown in Fig. 2a. It seems obvious that the branch points on $\mathfrak{M}$ may lie only at the point $i \bar{\zeta}=E$ where the Ernst equation is not valid.
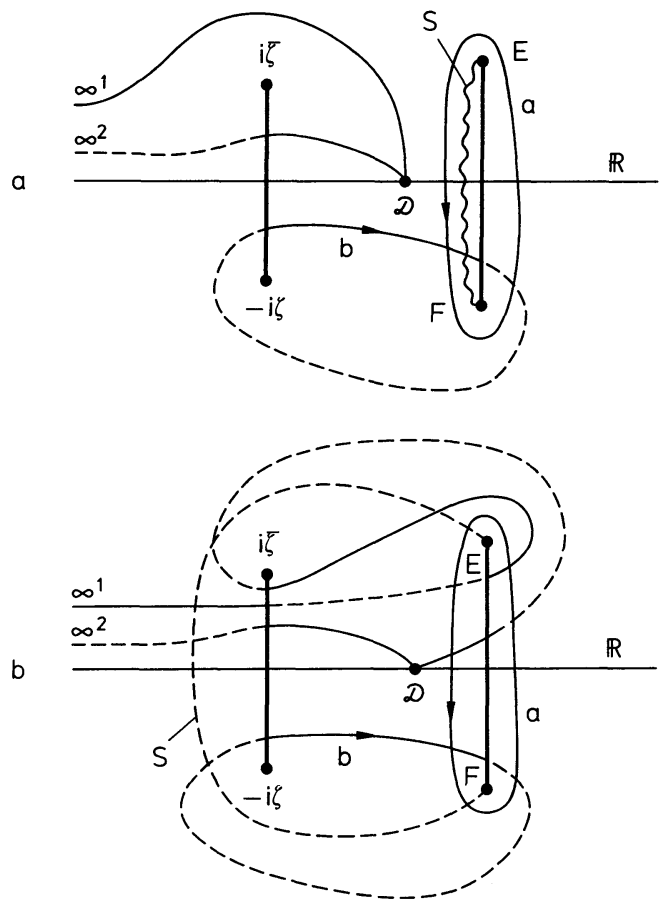
After a round of $i \bar{\zeta}$ about $E$ on $\mathscr{L}_{0}^{1}$ in a positive direction we obtain the curve $\mathscr{L}_{1}^{1}$ (Fig. 2b). This round leads to the following transformation of the variables setting the Ernst potential:

$$
\begin{gathered}
\mathbb{B}_{1}^{1}=\mathbb{B}_{0}^{1} ; \quad\left(U\left(\infty^{1}\right)-U(D)\right)_{1}^{1}=\left(U\left(\infty^{1}\right)-U(D)\right)_{0}^{1} ; \\
\left(U\left(\infty^{2}\right)-U(D)\right)_{1}^{1}=\left(U\left(\infty^{2}\right)-U(D)\right)_{0}^{1}+2 \mathbb{B}_{0}^{1} ; \\
\left.\left.W_{E F}(P)\right|_{\infty^{2}} ^{\infty}\right)_{1}^{1}=\left(\left.W_{E F}(P)\right|_{\infty} ^{\infty}\right)_{0}^{1} .
\end{gathered}
$$
that

Then using the periodicity properties of the theta-function it is easy to show

$$
\mathscr{E}_{1}^{1}(\varrho, z)=-e^{4 \pi \alpha} \mathscr{E}_{0}^{1}(\varrho, z) .
$$

So the points $E$ and $F$ are really branch points on $\mathfrak{M}$. Sheet $\mathfrak{M}_{1}^{1}$ seems to be "unphysical" because the Ernst potential tends to a negative limit $-e^{2 \pi \alpha}$ on its infinity.

If $\alpha=0$ then a double round of point $i \bar{\zeta}$ about $E$ leads on the initial sheet $\mathfrak{M}_{0}^{1}$. Therefore in this case our connection component $\mathfrak{M}^{1}$ consists of only two sheets: $\mathfrak{M}_{0}^{1}$ and $\mathfrak{M}_{1}^{1}$ (Fig. 3a) and $-\mathscr{E}_{0}^{1}=\mathscr{E}_{1}^{1}$; sheet $\mathfrak{M}_{0}^{1}$ seems to be "physical" and sheet $\mathfrak{M}_{1}^{1}$ "unphysical".

Case $\alpha \neq 0$ is more complex. If we round $j$ times about point $E$ then we arrive on sheet $\mathfrak{M}_{j}^{1}$ where

$$
\mathscr{E}_{j}^{1}=(-1)^{j} e^{4 \pi \alpha j} \mathscr{E}_{j}^{0} \quad(j \in \mathbb{Z}) .
$$

Hence in this case $\mathfrak{M}^{1}$ consists of an infinite number of sheets (Fig. 3b). Sheets with even numbers $\mathscr{M}_{2 k}^{1}$ are "physical"; they are connected with $\mathfrak{M}_{0}^{1}$ by some
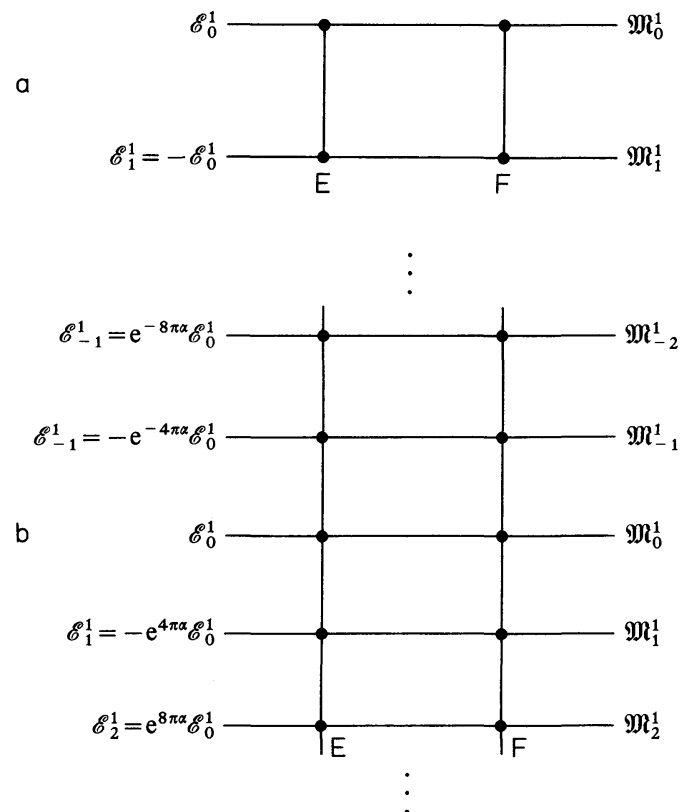

Fig. 3a and b. Topology of $\mathfrak{M}^{1}: a . \alpha=0, b . \alpha \neq 0$; Ernst potential on different sheets 
scaling transformation; sheets with the odd numbers $\mathfrak{M}_{2 k-1}^{1}$ seems to be "unphysical" because the Ernst potential is negative on their infinities.

So we described the topological structure of the connection component $\mathfrak{M}^{1}$ which include the sheet $\mathfrak{M}_{0}^{1}$ related to the curve $\mathscr{L}_{0}^{1}$ shown in Fig. $2 \mathrm{a}$.

Now it is necessary to make this description agree with the well-known topological structure of the Kerr-NUT solution which arises in the limit $E, F \rightarrow \gamma \in \mathbb{R}$. For this purpose it is necessary to consider another connection component $\mathfrak{M}^{2}$ of manifold $\mathfrak{M}$ starting from the curve $\mathscr{L}_{0}^{2}$ shown in Fig. 4. It is easy to verify that $\mathscr{E}_{0}^{2}=\left(\mathscr{E}_{0}^{1}\right)^{-1}$. If we round $j$ times about point $E$ then we arrive on sheet $\mathfrak{M}_{j}^{2}$, where $\mathscr{E}_{j}^{2}=\left(\mathscr{E}_{j}^{1}\right)^{-1}(j=0,1$ for $\alpha=0$ and $j \in \mathbb{Z}$ for $\alpha \neq 0)$.

If $E, F \rightarrow \gamma \in \mathbb{R}$ then curves $\mathscr{L}_{j}^{1,2}$ degenerate and a passage between $E$ and $F$ becomes impossible. Components $\mathfrak{M}^{1}$ and $\mathfrak{M}^{2}$ split on the separated sheets. Instead in this case we can continue potential $\mathscr{E}_{j}^{1}$ from $\mathfrak{M}_{j}^{1}$ to $\mathfrak{M}_{j}^{2}$ and obtain potential $\mathscr{E}_{j}^{2}=\left(\mathscr{E}_{j}^{1}\right)^{-1}$ (for any $j$ ). Therefore in this limit we obtain a family of nonconnected Kerr-NUT manifolds. In the case $\alpha=0$ it consists of two components the first is "physical" and the second seems to be "unphysical". The considered topological reconstruction in the case $\alpha=0$ is shown in Fig. 5a, b. In the case $\alpha \neq 0$

Fig. 4

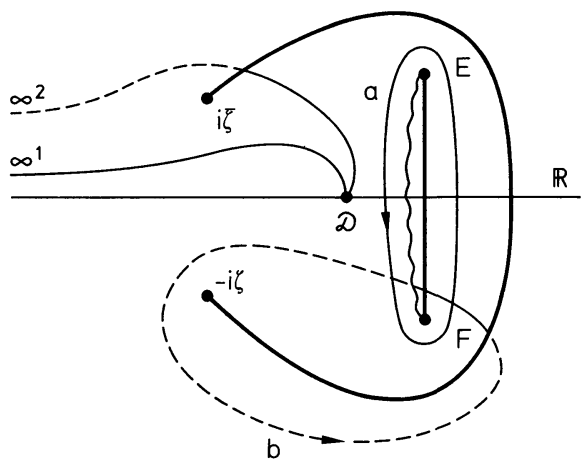

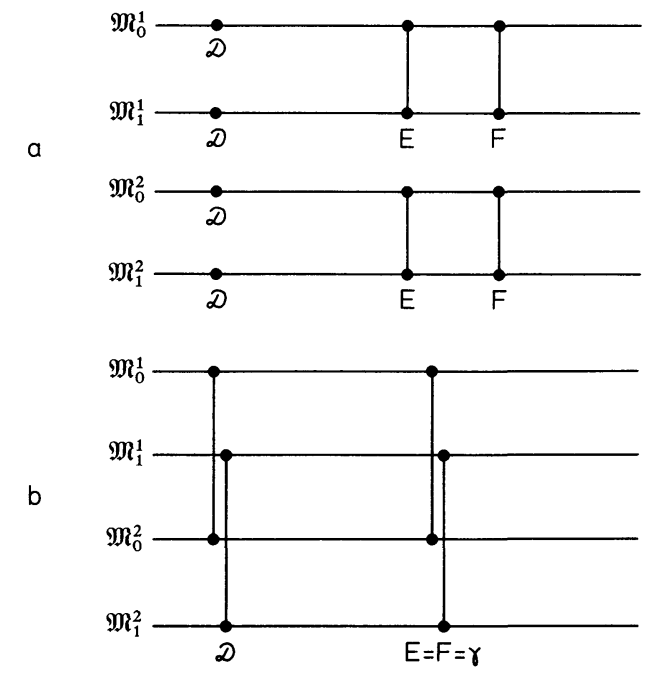

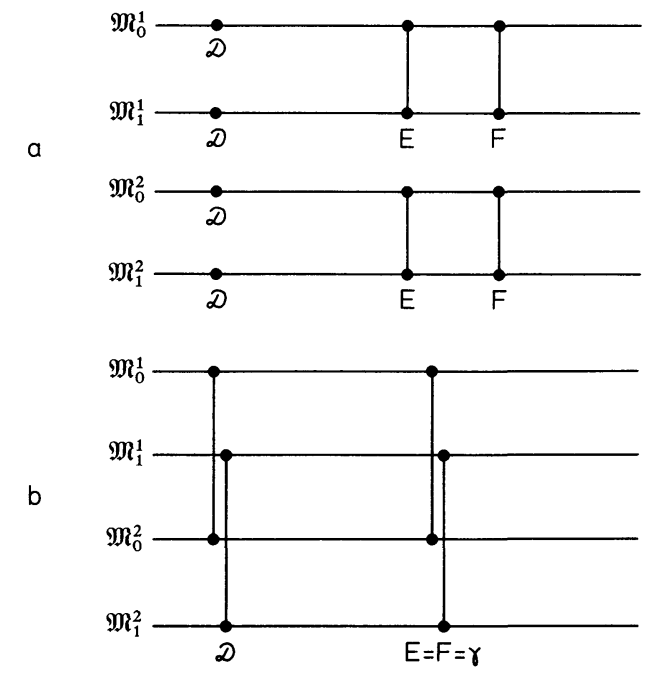

Fig. 5a and b 
one obtains an infinite family of similar "physcial" black holes and an infinite family of similar "unphysical" black holes.

It is rather interesting to describe in our terms [in Weyl coordinates $(\varrho, z)$ ], for example, the continuation from $\mathfrak{M}_{0}^{1}$ to $\mathfrak{M}_{0}^{2}$, i.e. the well-known continuation from the first sheet of the Kerr-NUT solution through the horizons on the second sheet. To obtain this continuation in our framework it is necessary to continuously deform $\mathscr{L}_{0}^{1}$ (with $E=F \equiv \gamma$ ) into $\mathscr{L}_{0}^{2}$. Note here that formula (1.5) may be used to provide not only stationary axisymmetric solutions but also non-stationary cylindrically-symmetric ones: it is sufficient to take $\varrho=i t$; then the moving branch cut $[z-t, z+t]$ on $\mathscr{L}$ lies on the real axis. This situation takes place between the horizons in the Kerr-NUT solution.

The way to deform $\mathscr{L}_{0}^{1}$ to $\mathscr{L}_{0}^{2}$ in (i.e. to glue $\mathfrak{M}_{0}^{1}$ and $\mathfrak{M}_{0}^{2}$ ) in the case $E=F=\gamma$ is represented in Figs. $6 \mathrm{a}-6 \mathrm{~g}$. Segment $[D, \gamma]$ plays the role of the horizons.

It is easy to see that the direct generalization of this procedure on the nondegenerated case is impossible; it seems that in this case the horizons are absent; the behaviour of the metric on the axis will be discussed in B.

\section{B. Behaviour of $\mathscr{E}(\zeta, \bar{\zeta})$ on Symmetry axis;}

\section{Mass, NUT-Parameter and Angular Momentum}

Let's consider a behaviour of genus one solution (in both: $E=\bar{F}$ and $E, F \in \mathbb{R}$ cases) on symmetry axis $(\varrho=0)$ of $\mathfrak{M}_{0}^{1}$. In this limit curve $\mathscr{L}_{0}^{1}$ (Fig. 2a) degenerates and it appears possible to find the dependence $\mathscr{E}(\varrho=0, z)$ in elementary functions. For this purpose we can take expressions (3.2) and (3.3) and to make substitutions:

a

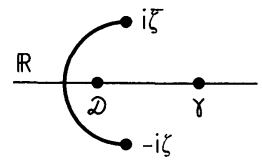

e

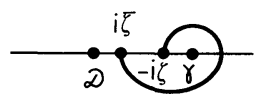

g

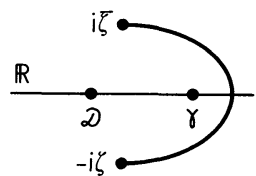

b

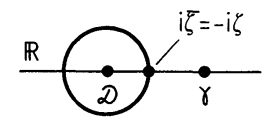

d

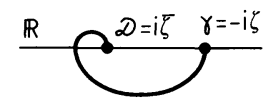

f

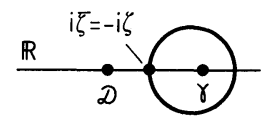

Fig. 6a-g. Deformation of $\mathscr{L}_{0}^{1}$ to $\mathscr{L}_{0}^{2}$ in the case $E=F \equiv \gamma$. Point on Kerr-NUT manifold is situated: a On the $1^{\text {st }}$ sheet outside of the event horizon. b On the event horizon. c-e Between the event and Cauchy horizons. f On Cauchy horizon. $g$ On the $2^{\text {nd }}$ sheet 
$D \rightarrow D, \gamma \rightarrow z, i \bar{\zeta} \rightarrow E,-i \zeta \rightarrow F$. Integral $W_{E F}$ will produce additional multiplication on $i e^{-2 \pi \alpha}$.

As a result one obtains:

$$
\mathscr{E}(\varrho=0, z)=i e^{-2 \pi \alpha} \frac{y-1+i e^{-2 \pi \alpha}(x+1)}{y+1+i e^{-2 \pi \alpha}(x-1)},
$$

where $\quad x=\frac{1}{D-z}[f(z)+f(D)], \quad y=\frac{1}{D-z}[f(z)-f(D)] \quad$ and $\quad f(\lambda) \equiv((\lambda-E)$ $\times(\lambda-F))^{1 / 2}$.

From (3.4) we can find the mass of our object on $\hat{\mathfrak{M}}_{0}^{1}$ :

$$
M_{0}=\frac{f(D)}{2}+\frac{i}{4}((E+F-2 D) \operatorname{ch} 2 \pi \alpha+2 f(D) \operatorname{sh} 2 \pi \alpha) .
$$

We see that the mass is complex, i.e. it consists of the ordinary mass $\operatorname{Re} M_{0}$ and the "magnetic mass" $\operatorname{Im} M_{0}$.

In the simplest case $\alpha=0$ (3.5) takes the following form:

$$
M_{0}=\frac{1}{2} \sqrt{(E-D)(F-D)}+\frac{i}{4}(E+F-2 D),
$$

i.e. the ordinary mass is half of the geometric average of $E-D$ and $F-D$ and the magnetic mass is half of the arithmetic average of $E-D$ and $F-D$. The presence of the magnetic mass is equivalent to the presence of the non-vanishing NUTparameter $\beta_{0}$ with $\operatorname{arctg} \beta_{0}=\operatorname{Re} M_{0} / \operatorname{Im} M_{0}$; when $\operatorname{Im} M_{0} \neq 0$ (or, equivalently, $\beta_{0} \neq 0$ ), the solution is asymptotically flat only in the weak sense. In some relations the role of the magnetic mass in relativity is equivalent to the role of magnetic charge in electrodynamics: the structure of geodesics in the field of magnetic mass is very similar to the structure of the trajectories of the charged particle in the field of the magnetic monopole [10].

The NUT-parameter may, of course, be omitted by the well-known Ehlers transformation; the resulting solution will have mass $M_{0}^{\prime}=\left|M_{0}\right|$. However, we shall not consider this possibility here.

To delete the NUT-parameter on $\mathfrak{M}_{0}^{1}$ it is necessary to require

$$
(E+F-2 D) \operatorname{ch} 2 \pi \alpha+2 \sqrt{(E-D)(F-D)} \operatorname{sh} 2 \pi \alpha=0
$$

or, equivalently,

$$
D=\frac{1}{2}(E+F) \pm \frac{i}{2}(E-F) \operatorname{sh} 2 \pi \alpha .
$$

It is easy to see that we can satisfy the condition (3.6) only in the case of a vertical immovable cut, i.e. $E=\bar{F}$. Indeed, in the case $E, F \in \mathbb{R}$ point $D$ given by (3.6) is complex (if $\alpha \neq 0$ ) that is impossible due to the reality of the pole divisor. If $\alpha=0$, one obtains $D=(E+F) / 2$; this is impossible too because $D$ must lie outside of the cut $[E, F]$.

We see that the case of the vertical immovable cut seems more physical; for this reason we considered in A the topological structure of the solutions from this class.

Using (3.4) we can directly compute the angular momentum of our object on $\hat{\mathfrak{M}}_{0}^{1}$ in the case of vanishing NUT-parameter when $E=\bar{F}$ and $D$ is set by (3.6):

$$
J_{0}=-(\operatorname{Im} E)^{2} \operatorname{ch} 2 \pi \alpha .
$$


The mass of this object is equal to $\operatorname{Re} M_{0}$ where $D$ is set by (3.6):

$$
M_{0}=\frac{1}{2}(\operatorname{Im} E) \operatorname{ch} 2 \pi \alpha .
$$

In the simplest case $\alpha=0$ we have

$$
M_{0}=\frac{1}{2} \operatorname{Im} E, \quad J_{0}=(\operatorname{Im} E)^{2} .
$$

As we shall see below, the factor $\operatorname{ch} 2 \pi \alpha$ may be considered as the density of our object.

\section{Singularities of the Ernst Potential: Case $E=\bar{F}$}

Here we shall consider the singularities of the Ernst potential: $\mathscr{E}=\infty$, because the rigorous analysis of the singularities of curvature is very complicated. However, for Kerr-NUT solutions these two types of singularities coincide; it is natural to suppose that this fact is also true for non-degenerated solutions.

Using well-known facts about the zeros of theta-function we immediately see that all points where $\mathscr{E}=\infty, 0$ may lie only at the point $i \bar{\zeta}=E$.

Let's consider, for example, the behaviour of $\mathscr{E}_{0}^{1}(\varrho, z)$ in the neighbourhood of the "branch ring" $i \bar{\zeta}=E$. To use in the limit $i \bar{\zeta} \rightarrow D$ the standard technique of the degeneration of finite-gap solutions (see, for example, $[1,2,8]$ ) one has to insert on $\mathscr{L}_{0}^{1}$ a new canonical basis of cycles $\left(a^{\prime}, b^{\prime}\right)=(b,-a)$ (cycles $a$ and $b$ are shown in Fig. 2a). In the new basis formula (3.1) takes the following form:

$$
\begin{aligned}
\mathscr{E}(\zeta, \bar{\zeta})= & \frac{\theta\left(U^{\prime}\left(\infty^{2}\right)-U^{\prime}(D)-K^{\prime}+\mathbb{B}^{\prime}(1 / 4+i \alpha)\right) \theta\left(U^{\prime}\left(\infty^{1}\right)-U^{\prime}(D)-K^{\prime}\right)}{\theta\left(U^{\prime}\left(\infty^{1}\right)-U^{\prime}(D)-K^{\prime}+\mathbb{B}^{\prime}(1 / 4+i \alpha)\right) \theta\left(U^{\prime}\left(\infty^{2}\right)-U^{\prime}(D)-K^{\prime}\right)} \\
& \times \exp \left\{\left.\left(2 i \alpha+\frac{1}{2}\right)\left(W_{E F}-\pi i U^{\prime}\right)\right|_{\infty 1} ^{\infty}\right\},
\end{aligned}
$$

where all shaded objects relate to the new basis $\left(a^{\prime}, b^{\prime}\right)$.

In the limit $i \bar{\zeta} \rightarrow E$ we have:

$$
\begin{gathered}
\mathbb{B}^{\prime} \underset{i \bar{\zeta} \rightarrow E}{\sim} \frac{2}{\pi i} \ln |i \bar{\zeta}-E|+O(1), \\
U^{\prime}\left(\infty^{1}\right)-U^{\prime}(D) \underset{i \widetilde{\zeta} \rightarrow E}{\sim} \frac{\mathbb{B}^{\prime}}{2}+O(1), \\
U^{\prime}\left(\infty^{2}\right)-U^{\prime}(D)_{i \zeta \rightarrow E} O(1) .
\end{gathered}
$$

Now using the definition of the theta-function we can apply the standard degeneration technique and obtain that the theta-function term in the previous formula for $\mathscr{E}$ is finite in our limit. For the exponential term we have:

$$
\begin{gathered}
\exp \left\{\left.\left(2 i \alpha+\frac{1}{2}\right)\left(W_{E F}-\pi i U^{\prime}\right)\right|_{\infty^{1}} ^{\infty^{2}}\right\} \underset{i \bar{\zeta} \rightarrow E}{\sim} \exp \left\{\left(2 i \alpha+\frac{1}{2}\right) \frac{\pi i \mathbb{B}^{\prime}}{2}\right\} \underset{i \tilde{\zeta} \rightarrow E}{\sim} \\
\sim|i \bar{\zeta}-E|^{1 / 2+2 i \alpha} O(1) \underset{i \bar{\zeta} \rightarrow E}{\longrightarrow} 0 .
\end{gathered}
$$


As a result we see

$$
\mathscr{E}_{j}^{1} \underset{i \bar{\zeta} \rightarrow E}{\longrightarrow} 0, \quad \mathscr{E}_{j} \underset{i \bar{\zeta} \rightarrow E}{\longrightarrow} \infty, \quad j=1,2(\alpha=0) \quad \text { or } \quad j \in \mathbb{Z}(\alpha \neq 0),
$$

i.e. the singularities of $\mathscr{E}$ on $\mathfrak{M}^{1}$ are absent and on $\mathfrak{M}^{2}$ they lie in the branch point $i \bar{\zeta}=E$. Note that the behaviour of $\mathscr{E}(\varrho, z)$ near $E$ completely agrees with the "branch role" of the ring $i \zeta=E$ on the Ernst manifold for our solution.

\section{Infinite Redshift Surface $\operatorname{Re} \mathscr{E}=0:$ Case $E=\bar{F}$}

The infinite redshift surface $\mathscr{S}$, where the metric coefficients are singular, is defined by the equation $g_{t t}=\operatorname{Re} \mathscr{E}=0$. From (3.4) we immediately see that this surface crosses the symmetry axis only at point $z=D$ on every sheet of $\mathfrak{M}$ (in the nondegenerated case). It is obvious that point $i \bar{\zeta}=E$ belongs to $\mathscr{S}$ too (on every sheet). In the degenerated case $E=F$ this surface crosses the axis at the points $z=D$ and $E=F$; from the topological point of view it is a sphere.

It appears possible to prove rigorously that in the case $\alpha=0, D=\operatorname{Re} \mathscr{E}$ the surface $\mathscr{S}$ has a form of a disk $z=\operatorname{Re} E, \varrho \leqq \operatorname{Im} E$ on any sheet of $\mathfrak{\mathfrak { M }}$. For example, let's prove this fact on $\mathfrak{M}_{0}^{1}$. If $z=\operatorname{Re} E$ and $\varrho \leqq \operatorname{Im} E$ then the curve $\mathscr{L}_{0}^{1}$ has a very symmetric form (Fig. 7) admitting an involution $\lambda \rightarrow-\lambda$. Using this involution we immediately obtain that

$$
U\left(\infty^{1}\right)-U(D)=\frac{\mathbb{B}}{2}, \quad U\left(\infty^{2}\right)-U(D)=-\frac{\mathbb{B}}{2}-\frac{1}{2} .
$$

Therefore $\mathscr{E}_{0}^{1}(z=\operatorname{Re} E, \varrho<\operatorname{Im} E)=i \frac{\theta(0)}{\theta(1 / 2)}$ and $\operatorname{Re} \mathscr{E}_{0}^{1}=0$ on our disc (Fig. 8a). It is obvious that this fact is true on every sheet of $\mathfrak{\mathfrak { M }}$.

Analogously we can prove that on the plane $z=\operatorname{Re} E$ outside of the disc $\varrho \leqq \operatorname{Im} E$ the Ernst potential is real.

When $D=\operatorname{Re} E$ but $\alpha \neq 0$ the form of $\mathscr{S}$ changes. It seems impossible to obtain the simple equation for the form of $\mathscr{S}$ in this case; we know only that it stretches the "branch ring" $i \bar{\zeta}=E$ and crosses the symmetry axis at $z=D$ (Fig. $8 \mathrm{~b}$ ) on every sheet of Ernst manifold. Then we see that on all sheets of Ernst manifold the surface $\mathscr{S}$ is a boundary between the "physical" sheets where $\operatorname{Re} \mathscr{E}>0$ and "unphysical" sheets where $\operatorname{Re} \mathscr{E}<0$.

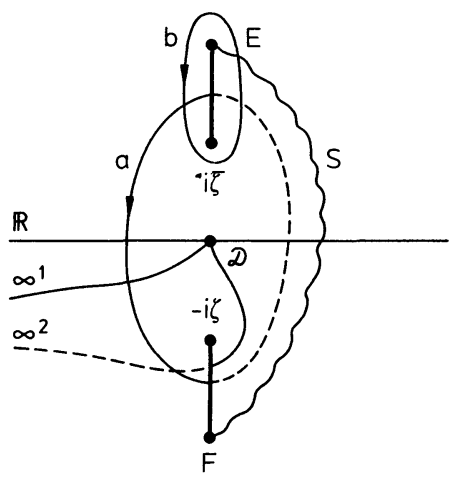


a

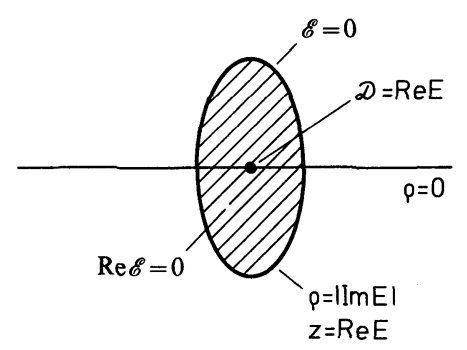

Fig. 8a and b

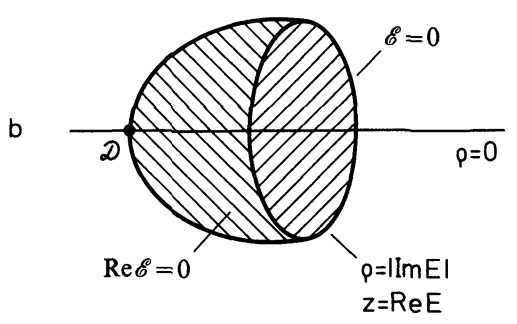

E. Behaviour of Metric on Symmetry Axis;

Structure of Einstein's Manifold: Case $E=\bar{F}$

Let's consider the behaviour of metric coefficients $A(\varrho, z)$ and $k(\varrho, z)$ set by $(1.2)$ on $\mathfrak{M}_{0}^{1}$. If we impose on $\mathfrak{M}_{0}^{1}$ the condition of asymptotic flatness then the coefficients $A$ and $k$ have to vanish on the infinity. From (1.2) we see that $A_{\zeta}(\varrho=0, z)=0$ and $k_{\zeta}(\varrho=0, z)=0$ in all points where $\operatorname{Re} \mathscr{E} \neq 0$ (in our case in all points of the symmetry axis excluding $z=D$ ). So vanishing of $k$ and $A$ on the infinity considered as initial conditions for the integral links (1.2) provides a regular behaviour of metric $(A \underset{e \rightarrow 0}{\longrightarrow} 0, k \underset{e \rightarrow 0}{\longrightarrow} 0)$ on the symmetry axis of $\hat{\mathfrak{M}}_{0}^{1}$. If we cross infinite redshift surface $\mathscr{S}$ then the behaviour of the metric becomes "unphysical."

Einstein's manifold in the case $\alpha \neq 0$ coincides with the Ernst manifold because in this case manifold $\hat{\mathfrak{M}}^{1}$ doesn't include non-trivial closed cycles. When $\alpha=0$ the $\hat{\mathfrak{M}}^{1}$ is a two-sheeted manifold and includes such cycles. Therefore in this case Einstein's manifold seems to be the infinite-sheeted covering of the Ernst manifold.

So the structure of Einstein's manifold seems to be the same for all $\alpha$. If starting from one of the "physical" sheets of this manifold we pass through the "branch ring" $i \bar{\zeta}=E j$ times then we arrive on the $j^{\text {th }}$ sheet; if $j$ is even then the new sheet appears "physical" too; if $j$ is odd then the new sheet appears "unphysical."

Of course, we discussed the continuation from one sheet to another only in terms of the Ernst potential although the rigorous analysis requires to consider this continuation in terms of the metric coefficients (particularly when we cross the infinite redshift surface). Therefore the statement about the unphysical character of the "odd" sheets of $\hat{\mathfrak{M}}^{1}$ is not quite rigorous and requires an additional consideration.

The previous treatment is true for both connection components considered in this section and marked by indexes 1 and 2 . However the component $\mathfrak{M}^{1}$ seems more "physical" in comparison with $\mathfrak{M}^{2}$ because on $\mathfrak{M}^{2}$ we have singularities of the 
Ernst potential on the "branch ring" and the mass of our object even on the "physical" sheets of $\mathfrak{M}^{2}$ is negative in contrast to $\mathfrak{M}^{1}$.

From (3.7) and (3.8) we see that in the case of the zero NUT-parameter the factor ch $2 \pi \alpha$ may be considered on $\mathfrak{M}_{0}^{1}$ as a density parameter of our massive rotating string-like object. However, the form of the infinite redshift surface depends essentially on $\alpha$ (Fig. 8): this form is simplest if $\alpha=0$ - the disc (Fig. 8a). In the case $\alpha \neq 0$ this surface seems to take a form of a "sack" (Fig. 8b).

\section{Concluding Remarks}

This paper is devoted to some initial steps in the clarification of the physical sense of algebraic-geometric solutions of stationary axisymmetric vacuum Einstein's equation found in [1-3]. We show that the asymptotic properties of these solutions coincide with the properties of degenerated-soliton solutions which describe the interaction of a few Kerr-NUT black holes on an arbitrary static background. Non-degenerated solutions describe an interaction of a few localized rotating genus one components on the static background. We consider in more detail a genus one solution on a flat background in the case of a vertical immovable branch cut; the opposite case of horizontal immovable branch cut seems less transparent and requires some additional consideration.

In the case of a vertical cut our genus 1 solution is set by four real parameters: divisor $D \in \mathbb{R}$, end of immovable branch cut $E \in \mathbb{C}$ and "density parameter" $\alpha \in \mathbb{R}$.

The most "physical" connection component of Einstein's manifold related to our solution consists topologically of an infinite number of $\mathbb{R}^{4}$ glued along a ring $z+i \varrho=E$. Half of these sheets are "physical"; others seems to be "unphysical." The mass of our object on one of the "physical" sheets is set by (3.5), i.e. consists of the real part - the ordinary mass, and the imaginary part - the magnetic mass responsible for the non-vanishing NUT-parameter. The infinite redshift surface seems to have in the general case a form of the "sack" (Fig. 8a) stretching the "branch ring" $\varrho=|\operatorname{Im} E|, z=\operatorname{Re} E$ on all sheets and crossing the symmetry axis at point $\varrho=0, z=D$; we proved rigorously that if $\alpha=0$ and $D=\operatorname{Re} E$ then it has a form of the disc. It divides the "physical" and "unphysical" sheets. The passage through the "branch ring" leads after the crossing of the infinite redshift surface from the "physical" to the "unphysical" sheet and vice versa.

For the NUT-parameter to be equal to zero the divisor $D$ has to satisfy condition (3.6); then one obtains a three-parametric solution which is asymptotically flat in a strong sense. The mass and angular momentum of this solution on $\mathfrak{M}_{0}^{1}$ are given by (3.8) and (3.7). The factor $\operatorname{ch} 2 \pi \alpha$ may be considered as a density parameter of the massive rotating string-like object: the form of the infinite redshift surface depends essentially on this factor (Fig. 8). In the simplest case $\alpha=0$ it has the form of a disc (Fig. 8a); in the case $\alpha \neq 0$ the probable form of this surface is a "sack" (Fig. 8b). Three parameters of our solution with a real positive mass play the role of the site on the axis, the radius and the density of the "string;" the angular rate is determined only by the radius of the "string" - for the equilibrium between the gravitational attraction and the centrifugal force.

Of course, the algebraic geometric solutions of gravity equations require further investigation. It is necessary to better understand the physical status of vacuum genus one solutions which were considered in this paper in both "horizontal" and "vertical" situations. It would be interesting also to analyze the 
properties of genus one solutions of the Einstein-Maxwell system [1,2] and to consider the effects of the interaction of few genus one objects.

It is a well-known fact that the manifolds of well-known algebraic geometric solutions of $\mathrm{KdV}$, NSch and others are dense in the manifolds of all almost periodic solutions of these equations. So we can suppose that the class of algebraic geometric solutions of Einstein's equation is dense in the class of all stationary axisymmetric asymptotically flat solutions. Then it is quite probable that the simplest genus one solution considered in this paper has some important physical sense.

Acknowledgements. I am grateful to Prof. V. B. Matveev and Prof. A. R. Its for their attention to this work. I thank Dr. G. A. Alexeev for very useful discussions.

\section{References}

1. Korotkin, D.A.: Finite-gap solutions of stationary axisymmetric vacuum Einstein equation. Teor. i Matem. Fisika. 77, 25-41 (1988)

2. Korotkin, D.A., Matveev, V.B.: Algebraic geometry of gravity equations. Algebra i Analis 1, 77-102 (1989) (English translation: Leningrad Mathematical Journal 1, 379-408 (1990))

3. Korotkin, D.A., Matveev, V.B.: Finite-gap integration of the equations having U-V pairs with a variable spectral parameter. In: Some topics on inverse problems. Sabatier, P. (ed.). Singapore: World Scientific, 1988

4. Novikov, S.P.: Periodic problem for KdV equation. Funct. Anal. Appl. 8, 54-66 (1974)

5. Dubrovin, B.A.: Inverse scattering problem for periodic finite-gap potentials. Funct. Anal. Appl. 9, 65-66 (1975)

6. Its, A.R., Matveev, V.B.: Finite-gap Hill's operators and multisoliton solutions of KdV equation. Theor. Math. Phys. 23, 51-64 (1975)

7. Korotkin, D.A.: Finite-gap solutions of $S U(2)$ and $S U(1,1)$ self-duality equations and their stationary axisymmetric reductions. Matem. Sbornik. 181, (7), 923-933 (1990)

8. Korotkin, D.A.: Self-dual Yang-Mills fields and deformations of algebraic curves. Commun. Math. Phys. 134, 397-412 (1990)

9. Neugebauer, G., Kramer, D.: Einstein-Maxwell solitons. J. Phys. A 16, 1927-1936 (1983)

10. Zimmerman, R.L., Shamir, B.Y.: Geodesics for the NUT metric and gravitational monopoles. Gen. Rel. Grav. 21, 821-848 (1989)

11. For reviews of the exact solutions of Einstein's equations, see:

Kramer, D., Stephani, H., MacCallum, M., Herlt, E.: Exact solutions of the Einstein's field equations. Berlin: V.E.B., 1980;

Alexeev, G.A.: Exact solutions in general relativity: Trudy MIAN 176, 211-258 (1987)

Communicated by N. Yu. Reshetikhin 